Brit. J. vener. Dis. (1958), 34, 83.

\title{
RESULTS OF LOCAL CORTISONE THERAPY IN SYPHILITIC INTERSTITIAL KERATITIS*
}

\author{
BY \\ A. N. ASHWORTH \\ Royal Eve Hospital, Manchester
}

It is just under 10 years ago since the effect of cortisone on inflammatory diseases of the eye was first reported by Hench, Kendall, Slocumb, and Polley (1949). During the ensuing years a vast number of reports and papers have been published concerning its application in almost every known eye condition. By 1951 some idea of its limitations was beginning to emerge (Duke Elder, 1951). Its principal application was in suppressing exudation and increased vascularity in certain inflammatory eye diseases. These effects could be obtained by local application in diseases of the anterior segment of the eye, and by systemic application in diseases of the posterior segment. This statement which emerged as a result of the publications of Duke Elder (1951) and Woods $(1951 \mathrm{a}, \mathrm{b})$ has never been contradicted and as a broad principle still holds true. It was known that hormone therapy suppressed the inflammatory response and did not produce a cure of any particular condition, and that it should be used as an adjuvent only to accepted therapy. It was questioned whether it was a good thing to suppress the inflammatory response, and caution in assessing the ultimate value of cortisone treatment was expressed. In 1951 the drug had only been available in limited quantities for about 18 months, and ophthalmic literature was full of reports of the treatment of eye diseases by cortisone, all obviously short-term reports. Few of these cases have been reported again from the point of view of long-term assessment. My own experience is that some of the cases which I assessed in 1954 still remain with me as patients (Ashworth, 1954). The trend in the literature then followed the development of fresh derivitives of the corticosteroids, such as hydrocortisone, prednisone, and prednisolone, and fresh reviews of cases treated by these drugs were published. These developments were reviewed by Grant (1957).

* Paper read to M.S.S.V.D. at Manchester, March 29, 1958. Received for publication April 2, 1958.
My own view is that there is a definite indication for cortisone therapy in three eye conditions only:

(1) Sympathetic ophthalmia

(2) Interstitial keratitis

(3) Acne keratitis

It is with the second of these indications that I am now concerned.

Five years ago I reported upon a series of 28 cases of interstitial keratitis, which had been treated over the previous 2 years (Ashworth, 1953). The initial stages of the disease constitute a period of acute misery for the patient, and a dramatic relief was found, without exception, in all cases. It was said at that time that although the immediate effects were good, the ultimate effects might not: be so good, and that the patients might remain subject to later relapses of the condition. In addition, doubt was expressed that the ultimate results in terms of visual acuity would be any better than those obtained by other forms of treatment. The principal source of these doubts came from a paper by Woods (1951). He stated that the stage of vascularization of the cornea was essential in the development of immunity, and ultimately in the prevention of later relapses in cases of interstitial keratitis. The literature was critically reviewed from this standpoint by Horne (1955), who also offered an explanation for the failures of treatment reported by some authors. The main reasons for failure seemed to be that the dosage of cortisone was inadequate or that the duration of the course and the period of follow-up were too short.

Whenever doubt of the long-term value of cortisone therapy has been expressed, no time interval has been suggested at which a final assessment could be made. Horne's series of cases assessed up to 3 years after commencing cortisone therapy contains the longest follow-up period that I have been able to find. 
It was therefore decided to review my original 28 patients, together with some others, after the lapse of 5 years. This paper discusses the results of treatment in fifty patients, with 84 eyes affected, most of whom have been under observation for 5 years or more, and to many of whom no treatment has been given for the past 5 years.

The principal points of interest are the following:

(1) Corneal vascularization and the relationship of this to the occurrence of relapses after discontinuing treatment;

(2) The number of relapses occurring during treatment;

(3) The results of treatment in terms of visual acuity.

\section{Method of Treatment}

Topical treatment was employed in all cases. The drug is not absorbed systemically from local therapy, so there is no risk of side-effects however long the local treatment is maintained. The method of using cortisone is by drops, or by ointment, or by a combination of both. Initial treatment is frequent (2-hourly) until the eye becomes white and comfortable. This happens within 4 to 5 days, and treatment is then given 4-hourly. If a case is severe and is not settling in 4 to 5 days, a subconjunctival injection (10 mg. in $0.4 \mathrm{ml}$.) is given in addition. This method of treatment will control all cases, and the injection, which is painful for the patient, does not usually need to be repeated. It is also necessary to keep the pupil dilated with atropine, which is used initially 4-hourly until the eye is controlled; it may then be used twice daily until the eye has been quiet for about 2 weeks, when it may be discontinued unless there is any tendency for the eye to relapse, when it must be used again.

The majority of the cases in this series were treated by cortisone acetate suspension in normal saline $(5 \mathrm{mg} . / 1 \mathrm{ml}$.) or cortisone ointment $(15 \mathrm{mg}$. $/ 1 \mathrm{~g}$.). Hydrocortisone acetate $(5 \mathrm{mg} . / 1 \mathrm{ml}$.)was also used, but prednisone acetate $(0.25$ per cent.) and prednisolone free alcohol $(0 \cdot 25$ per cent.), which are reported to have three or four times the activity of cortisone and hydrocortisone by Gordon (1956), were not available for local use in our clinic. It would be advantageous to use these substances in treating interstitial keratitis, since I think it important to get the maximum possible concentration of hormone into the eye as soon as possible. Prednisone and prednisolone pass easily into the anterior chamber in topical application (Leopold, Kruman, and Green, 1955), and would therefore be useful in helping to control the anterior uveitis which invariably accompanies interstitial keratitis.
The only local complication which was found in this series was the occasional occurrence of styes (blepharitis). These are easily controlled by the use of simple treatment with albucid, and in view of this I do not think it any advantage to use combined cortisone and antibiotics over such long courses of treatment.

\section{Case Management}

During the initial stages of treatment our patients are treated as in-patients, which is advisable for two reasons:

(1) Whilst controlling interstitial keratitis in its early stages, it is important that the cortisone should be used in adequate quantities and the treatment carried out at regular and frequent intervals. This is best achieved with the patient under supervision.

(2) A daily injection of 1 million units of penicillin intramuscularly for 10 days is also given. This is more convenient with in-patients, especially children.

Within 5 days the pain, irritation, and photophobia will be disappearing and in 10 to 14 days it is usually possible to discharge the case on 4-hourly treatment, with a weekly follow-up. This ophthalmic follow-up is carried out at the same time as the attendance at the V.D. clinic and by this means regular supervision is ensured. The cortisone is continued during the day 4-hourly for 4 weeks and is then gradually reduced to 8-hourly, and then to twice daily. At any time whilst the dosage is being reduced, a relapse or escape reaction may manifest itself. This is recognized by the patient himself by a return of irritation and photophobia. So long as the symptoms are absent, the eye remains white and the condition is under control and will gradually resolve. As soon as it has been possible to reduce treatment to a twice daily routine and to maintain this for 4 weeks, cortisone may be stopped altogether. At this stage the patient should be seen frequently for the keratitis will recur if it has not "burned itself out". Then a further period of cortisone and possibly atropine must be given to control it.

In answer to the question-"How long is it necessary to continue cortisone?" the cessation of therapy may be used as a "test of cure". If there is no return of symptoms within 3 months, treatment has been adequate and the chances of a relapse later than this are small. A relapse occurring under these conditions is quickly controlled and does no damage to the eye so far as sight is concerned. I do not think there is any other way of recognizing 
whether treatment has been given for long enough, since with adequate therapy all signs and symptoms are obliterated by the cortisone.

\section{Material}

The fifty patients ( 84 eyes) which form the basis of this paper comprise all the cases which became available for treatment in one hospital from 1951 to 1956 (6 years). An analysis of the year in which patients came for treatment shows that the majority attended during the earlier years of the investigation, which indicates that the incidence of interstitial keratitis has decreased (Table I).

TABLE I

YEAR IN WHICH PATIENTS BEGAN TREATMENI

\begin{tabular}{c|c|c|c|c|c|c|c}
\hline Year .. .. & 1951 & 1952 & 1953 & 1954 & 1955 & 1956 & Total \\
\hline $\begin{array}{c}\text { No. of Cases } \\
\text { commencing }\end{array}$ & 7 & 21 & 7 & 9 & 5 & 1 & 50 \\
\hline
\end{tabular}

The majority of these patients were under observation for 4 years or more, and it was found that they could all be controlled within a short time, but that, once under control, they would relapse if therapy was stopped before the condition had resolved itself.

According to Woods (1951) a relapse may occur at some unspecified later date, if adequate immunity has not been built up in the affected cornea. He also states that the prevention of corneal vascularization increases the tendency to these late relapses. I do not believe that this is so, and it has not been borne out in my experience.

In Table II the patients are classified according to the incidence of relapses occurring during treatment.

TABLE II

DISTRIBUTION OF CASES BY INCIDENCE OF RELAPSES

\begin{tabular}{|c|c|c|c|c|}
\hline \multicolumn{3}{|c|}{ Group } & No. of Cases & No. of Eyes \\
\hline 1. & No relapses & . & 24 & 38 \\
\hline 2. & Relapsing course .. & $\cdots$ & 9 & 16 \\
\hline 3. & Relapse within 3 months & $\cdots$ & 6 & 10 \\
\hline 4. & $\begin{array}{c}\text { Late relapse after more } \\
\text { months .. }\end{array}$ & $\begin{array}{l}3 \\
\cdots\end{array}$ & 5 & 10 \\
\hline 5. & Defaulters... & . & 4 & 6 \\
\hline
\end{tabular}

Group 1.- These patients (Table A) had no relapse at any time, and all had rapid relief of symptoms. Control was maintained for times varying from 2 to 12 months and in one case for 24 months. During this time irritation and photophobia were absent, the patient was able to get about, attend school, or work, and corneal opacity, keratic precipitates, and corneal vessels all gradually disappeared. Most of these patients have been assessed 5 years after treatment was discontinued, and I do not think it likely that any of them will relapse in the future. 28 eyes had no corneal vessels at any stage, 10 eyes had corneal vessels in varying degrees from slight to severe. It appears to make no difference to the eventual outcome of the case whether corneal vessels are present or not (Table III).

TABLE III

RESULTS OF TREATMENT IN GROUP I (38 EYES)

\begin{tabular}{|c|c|c|c|c|c|}
\hline \multirow{2}{*}{ State of Cornea } & \multirow{2}{*}{$\begin{array}{c}\text { Duration of } \\
\text { Treatment } \\
\text { (mths) }\end{array}$} & \multicolumn{3}{|c|}{ Visual Acuity } & \multirow{2}{*}{$\begin{array}{l}\text { Total } \\
\text { No. of } \\
\text { Eyes }\end{array}$} \\
\hline & & $6 / 6-6 / 9$ & $6 / 12$ & $6 / 24$ & \\
\hline $\begin{array}{c}\text { Corneal vessels } \\
\text { present }\end{array}$ & $2-9$ & 8 & 一 & 2 & 10 \\
\hline No corneal vessels .. & $1-12$ & 27 & 1 & - & 28 \\
\hline $\begin{array}{ccc}\text { Corneal scar remain- } \\
\text { ing } & . . & \ldots\end{array}$ & $3-24$ & 2 & 1 & 2 & 5 \\
\hline
\end{tabular}

Case 12, which has been assessed 5 years after stopping local treatment, was the most severe case I have ever seen, with bilateral central corneal necrosis, the so-called corneal gumma. I am sure this patient would now be blind but for cortisone therapy.

Group 2.- These patients (Table B) relapsed within a few days, when treatment was reduced or discontinued, and did so because treatment was inadequate, or had been stopped too soon. Control was rapidly regained by resumption of the treatment. In this type of case it is necessary to persist with treatment until no further relapses occur. Most of these patients have been assessed 3 years after all treatment was discontinued. The eventual visual result is good in all cases (Table IV). The duration of

TABLE IV

RESULTS OF TREATMENT IN GROUP 2 (16 EYES)

\begin{tabular}{l|c|c|c|c}
\hline \multicolumn{1}{c|}{ State of Cornea } & $\begin{array}{c}\text { Duration of } \\
\text { Treatment } \\
\text { (mths) }\end{array}$ & \multicolumn{2}{|c|}{ Visual Acuity } & $\begin{array}{c}\text { Total } \\
\text { No. of } \\
\text { Eyes }\end{array}$ \\
\hline Corneal vessels present & $1-20$ & 7 & - & 7 \\
\hline No corneal vessels ... & $6-66$ & 8 & 1 & 9 \\
\hline Corneal scar remaining & $10-66$ & 5 & - & 5 \\
\hline
\end{tabular}

treatment does not affect the eventual visual result, although the incidence of corneal scar was higher. One eye with 6/36 visual acuity developed an optic nerve atrophy in spite of systemic penicillin therapy. A larger proportion of these patients had corneal vascularization. 
Group 3.-These six cases (Table C) are included because they relapsed within 3 months of discontinuing cortisone therapy, because treatment had been stopped too soon. Only two affected eyes had corneal vascularization. The interval between stopping treatment and assessment is about 4 years. The visual acuity obtained was $6 / 6$ in all cases and no corneal scarring occurred (Table V).

TABLE V

RESULTS OF TREATMENT IN GROUP 3 (10 EYES)

\begin{tabular}{cc|c|c}
\hline State of Cornea & & $\begin{array}{c}\text { Visual Acuity } \\
6 / 6-6 / 9\end{array}$ & $\begin{array}{c}\text { Total No. } \\
\text { of Eyes }\end{array}$ \\
\hline Corneal vessels present $\ldots$ & $\ldots$ & 2 & 2 \\
\hline No corneal vessels present & $\ldots$ & 7 & 7 \\
\hline Corneal scar remaining.. & $\ldots$ & 0 & 0 \\
\hline
\end{tabular}

Group 4.-These patients (Table D) had "late" relapses more than 3 months after discontinuing treatment (Table VI). Only three out of ten eyes had corneal vascularization. The control of the relapses was immediate and the eventual visual result was $6 / 6$ in all cases. Most of these patients have been assessed 5 years after discontinuing treatment. The intervals between attacks varied from 4 months to 4 years.

TABLE VI

RESULTS OF TREATMENT IN GROUP 4 (10 EYES)

\begin{tabular}{cc|c|c}
\hline State of Cornea & $\begin{array}{c}\text { Visual Acuity } \\
6 / 6-6 / 9\end{array}$ & $\begin{array}{c}\text { Total No. } \\
\text { of Eyes }\end{array}$ \\
\hline Corneal vessels present $\ldots$ & $\ldots$ & 3 & 3 \\
\hline No corneal vessels & $\ldots$ & $\ldots$ & 8 \\
\hline Corneal scar remaining $\ldots$ & $\ldots$ & 1 & 8 \\
\hline
\end{tabular}

Group 5.--In these four patients the inflammation was immediately controlled by cortisone (Table E).
They continued to attend for varying lengths of time but then failed to come for further treatment. Recent attempts to persuade them to attend for a final assessment produced no results, and they are omitted from the final analysis.

Group 6.-When the corneal haze cleared in two patients each with both eyes affected, they were both seen to have well-established diffuse choroiditis (Table F).

Systemic cortisone was not given, and local cortisone controlled the keratitis and anterior uveitis. However systemic penicillin and local cortisone failed to control the posterior uveitis or choroiditis, which eventually gave a bad visual result. Similar cases have been reported by Macfarlane (1957).

\section{Conclusions}

All types of interstitial keratitis, whether treated immediately or within a few weeks of the onset of the condition, whether the cornea is vascularized or not, can be controlled very quickly, and the patient's symptoms are relieved. The control can be maintained by adequate local cortisone therapy, adequately supervised, for an indefinite period until the condition has died out and all signs in the cornea and anterior uveal tract have disappeared. Lesions of the posterior uveal tract, however, are unaffected by local treatment. Short-term relapses occur frequently but are all easily controlled again and do not affect the eventual prognosis. Long-term relapses more than 3 months after treatment had been discontinued occurred in only five cases, and these were all controlled quickly with a good final result (Table VII).

A visual acuity of $6 / 6$ or $6 / 9$ was obtained in 71 eyes $-6 / 12$ in one eye (due to corneal scarring), $6 / 18$ in one eye, and 6/24 in one eye (also due to corneal scar). In four eyes the visual result was poor because of choroiditis, and in one because of optic

TABLE VII

RESULTS OF TREATMENT IN ALL SIX GROUPS

\begin{tabular}{|c|c|c|c|c|c|c|c|c|c|c|c|}
\hline \multirow{3}{*}{ Group } & \multirow{3}{*}{ No. of Cases } & \multirow{3}{*}{ No. of Eyes } & \multicolumn{8}{|c|}{ Visual Acuity } & \multirow{3}{*}{ Remarks } \\
\hline & & & \multicolumn{2}{|c|}{$6 / 6-6 / 9$} & \multicolumn{2}{|c|}{$6 / 12$} & \multicolumn{2}{|c|}{$6 / 18$} & \multicolumn{2}{|c|}{$6 / 24$} & \\
\hline & & & No. & $\%$ & No. & $\%$ & No. & $\%$ & No. & $\%$ & \\
\hline 1 & 24 & 38 & 37 & 97 & $1^{*}$ & 3 & & & & & ${ }^{*}$ due to corneal scar. \\
\hline 2 & 9 & 16 & 15 & 94 & & & $1^{*}$ & 6 & & & ${ }^{*}$ due to corneal scar. \\
\hline 3 & 6 & 10 & 10 & 100 & & & & & & & \\
\hline 4 & 5 & 10 & 10 & 100 & & & & & & & \\
\hline 6 & 2 & 4 & & & & & 1 & 25 & & & $\begin{array}{l}\text { Three eyes (75\%) less than } 3 / 60 \text { due } \\
\text { to Choroiditis. }\end{array}$ \\
\hline
\end{tabular}


atrophy. At the final examination all the affected eyes had a freely mobile pupil, with no posterior synechiae and no visible signs of anterior uveal damage.

\section{Summary}

84 eyes affected with interstitial keratitis in fifty patients have been treated locally with cortisone.

Such treatment gives freedom from symptoms, a good expectation of a $6 / 6$ visual acuity, and no corneal scarring, or so little that it does not interfere with visual acuity.

The presence of corneal vascularization bears no relationship to the good prognosis, or the incidence of relapses, or the eventual outcome of the case.
In the majority of cases reported all treatment was discontinued 5 years ago, and they were all re-examined during February, 1958.

\section{REFERENCES}

Ashworth, A. N. (1953). Brit. J. vener. Dis., 29, 3.

Duke-Elder, S., Duthie, O. M., Foster, J., Law, F. W., Lister, A. Reid, A. M., Scott, G. I., Zorab, E. C., and Goldsmith, A. J. B. (1951). Brit. J. Ophthal., 35, 672.

Gordon, D. M (1956). Amer. J Ophthal, 41, 593.

Grant, W. M. (1957). A.M.A. Arch. Ophthal., 58, 265.

Hench, P. S., Kendall, E. C., Slocumb, C. H., and Polley, H. F (1949). Proc. Mayo Clin., 24, 181.

Horne, G. O. (1955). Brit. J. vener. Dis., 31, 9.

Leopold, I. H., Kruman, H. S., and Green, H. (1955). Trans. Amer. Acad. Ophthal. Otolaryng., 59, 771.

MacFarlane, W. V. (1957). Brit. J vener Dis. 33, 165.

Woods, A. C. (1951a). Amer. J. Ophthal., 34, 945.

(1951b). Amer. J. Syph., 35, 517.

\section{APPENDIX}

TABLE A

GROUP 1: 24 CASES TREATED WITHOUT RELAPSES

\begin{tabular}{|c|c|c|c|c|c|c|c|c|c|c|c|c|}
\hline $\begin{array}{l}\text { Case } \\
\text { No. }\end{array}$ & $\begin{array}{c}\text { Age } \\
\text { (yrs) }\end{array}$ & Sex & $\begin{array}{c}\text { Eye } \\
\text { Involved }\end{array}$ & $\begin{array}{c}\text { Duration } \\
\text { of } \\
\text { Symptoms } \\
\text { (wks) }\end{array}$ & $\begin{array}{c}\text { Interval } \\
\text { between } \\
\text { Onset in } \\
\text { Two Eyes } \\
\text { (wks) }\end{array}$ & $\begin{array}{c}\text { Date of } \\
\text { Starting } \\
\text { Treatment }\end{array}$ & $\begin{array}{c}\text { Duration } \\
\text { of } \\
\text { Treatment } \\
\text { (mths) }\end{array}$ & $\begin{array}{l}\text { Corneal } \\
\text { Vessels }\end{array}$ & $\begin{array}{l}\text { Interval between } \\
\text { Stopping } \\
\text { Treatment and } \\
\text { Assessment } \\
\text { (yrs) }\end{array}$ & $\begin{array}{c}\text { Visual } \\
\text { Acuity } \\
\text { (corrected) }\end{array}$ & $\begin{array}{l}\text { Corneal } \\
\text { Opacity }\end{array}$ & $\underset{\text { Reaction }}{\text { Wassermann }}$ \\
\hline \multirow{2}{*}{3} & \multirow{2}{*}{16} & \multirow{2}{*}{$\mathbf{M}$} & $\mathbf{R}$ & 3 & & 3.51 & 1 & - & 7 & $6 / 6$ & \multirow[t]{2}{*}{-} & \multirow{2}{*}{+} \\
\hline & & & $\mathrm{L}$ & 2 & & 3.51 & 1 & + & 7 & $6 / 6$ & & \\
\hline \multirow{2}{*}{5} & \multirow{2}{*}{11} & \multirow{2}{*}{ F } & $\mathbf{R}$ & 4 & \multirow{2}{*}{4} & 6.51 & 3 & - & $6 \frac{1}{2}$ & $6 / 6$ & & \multirow{2}{*}{+} \\
\hline & & & $\mathrm{L}$ & 4 & & 7.51 & 3 & - & $6 \frac{1}{2}$ & $6 / 6$ & & \\
\hline \multirow{2}{*}{7} & \multirow{2}{*}{4} & \multirow{2}{*}{$\mathbf{F}$} & $\mathbf{R}$ & 2 & \multirow{2}{*}{32} & 8.51 & 3 & - & 6 & $6 / 6$ & Faint & \multirow{2}{*}{+} \\
\hline & & & $L$ & 2 & & 1.52 & 4 & - & 6 & $6 / 6$ & & \\
\hline 8 & 17 & $\mathbf{M}$ & L & 2 & & 10.51 & 2 & + & 6 & $6 / 6$ & & + \\
\hline \multirow{2}{*}{9} & \multirow{2}{*}{13} & \multirow{2}{*}{ F } & $\mathbf{R}$ & 4 & \multirow{2}{*}{6} & 1.52 & 12 & - & 5 & $6 / 6$ & & \multirow{2}{*}{+} \\
\hline & & & L & 2 & & 3.52 & 10 & & 5 & $6 / 6$ & Faint & \\
\hline \multirow{2}{*}{10} & \multirow{2}{*}{13} & \multirow{2}{*}{$\mathbf{M}$} & L & 4 & & 12.51 & 5 & + & $5 \underline{b}$ & $6 / 9$ & & \multirow{2}{*}{+} \\
\hline & & & $\mathbf{R}$ & 4 & & 1.52 & 4 & & $5 \underline{1}$ & $6 / 9$ & & \\
\hline \multirow{2}{*}{12} & \multirow{2}{*}{12} & \multirow{2}{*}{$\mathbf{F}$} & $\mathbf{R}$ & 8 & \multirow{2}{*}{2} & 1.52 & 12 & + & 5 & $6 / 24$ & Marked & \multirow{2}{*}{+} \\
\hline & & & L & 8 & & 2.52 & 12 & + & 5 & $6 / 18$ & Marked & \\
\hline \multirow{2}{*}{13} & \multirow{2}{*}{6} & \multirow{2}{*}{$\mathbf{F}$} & $\mathbf{R}$ & 3 & 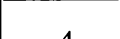 & 2.52 & 7 & - & $5 \frac{1}{2}$ & $6 / 6$ & & 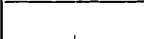 \\
\hline & & & L & 2 & 4 & 3.52 & 6 & 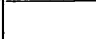 & $5 \frac{1}{2}$ & $6 / 6$ & & + \\
\hline 10 & 18 & $F$ & $\mathbf{R}$ & 4 & & 5.52 & 12 & & 5 & $6 / 6$ & & \\
\hline 19 & 10 & $\mathbf{r}$ & L & 4 & & 5.52 & 12 & - & 5 & $6 / 6$ & & \\
\hline 20 & 26 & $F$ & $\mathbf{R}$ & 6 & & 8.52 & 12 & - & $4 \frac{1}{2}$ & $6 / 6$ & & + \\
\hline 22 & 22 & $\mathbf{M}$ & L & 4 & & 9.52 & 4 & + & 5 & $6 / 6$ & & + \\
\hline 23 & 7 & $\mathbf{M}$ & $\mathbf{R}$ & 4 & & 10.52 & 5 & - & 5 & $6 / 6$ & & + \\
\hline 24 & 10 & $\mathbf{M}$ & L & 5 days & & 10.52 & 12 & - & $4 \frac{1}{2}$ & $6 / 6$ & & + \\
\hline
\end{tabular}


TABLE A-continued

GROUP 1: 24 CASES TREATED WITHOUT RELAPSES

\begin{tabular}{|c|c|c|c|c|c|c|c|c|c|c|c|c|}
\hline $\begin{array}{l}\text { Case } \\
\text { No. }\end{array}$ & $\begin{array}{l}\text { Age } \\
(\mathrm{yrs})\end{array}$ & Sex & $\begin{array}{c}\text { Eye } \\
\text { Involved }\end{array}$ & $\begin{array}{c}\text { Duration } \\
\text { of } \\
\text { Symptoms } \\
\text { (wks) }\end{array}$ & $\begin{array}{c}\text { Interval } \\
\text { between } \\
\text { Onset in } \\
\text { Two Eyes } \\
\text { (wks) }\end{array}$ & $\begin{array}{l}\text { Date of } \\
\text { Starting } \\
\text { Treatment }\end{array}$ & $\begin{array}{c}\text { Duration } \\
\text { of } \\
\text { Treatment } \\
\text { (mths) }\end{array}$ & $\begin{array}{l}\text { Corneal } \\
\text { Vessels }\end{array}$ & $\begin{array}{l}\text { Interval between } \\
\text { Stopping } \\
\text { Treatment and } \\
\text { Assessment } \\
\text { (yrs) }\end{array}$ & $\begin{array}{c}\text { Visual } \\
\text { Acuity } \\
\text { (corrected) }\end{array}$ & $\begin{array}{l}\text { Corneal } \\
\text { Opacity }\end{array}$ & $\begin{array}{c}\text { Wassermann } \\
\text { Reaction }\end{array}$ \\
\hline \multirow{2}{*}{25} & \multirow{2}{*}{8} & \multirow{2}{*}{$\mathbf{M}$} & $\mathbf{R}$ & 1 & \multirow{2}{*}{24} & 11.52 & 9 & + & $4 !$ & $6 / 6$ & & \\
\hline & & & L & 2 & & 5.53 & 4 & & $4 ! \frac{1}{2}$ & $6 / 6$ & & \\
\hline 26 & 5 & $\mathbf{F}$ & $\mathbf{L}$ & 5 days & & 3.53 & 7 & - & $4 !$ & $6 / 6$ & & $\%$ \\
\hline \multirow{2}{*}{31} & \multirow{2}{*}{8} & \multirow{2}{*}{$\mathbf{F}$} & $\mathbf{R}$ & 2 & \multirow{2}{*}{1} & 7.53 & 2 & - & $4 !$ & $6 / 6$ & & \multirow{2}{*}{+} \\
\hline & & & L & 2 & & 7.53 & 2 & - & $4 !$ & $6 / 6$ & & \\
\hline 33 & 16 & $\mathbf{F}$ & L & 3 & & 10.53 & 6 & - & 4 & $6 / 6$ & & + \\
\hline 35 & 7 & $\mathbf{F}$ & $\mathbf{R}$ & 2 & & 3.54 & 4 & + & 4 & $6 / 6$ & & + \\
\hline 36 & 7 & $\mathrm{~F}$ & $\mathbf{R}$ & 2 & & 3.54 & 4 & + & 4 & $6 / 6$ & & + \\
\hline \multirow{2}{*}{39} & \multirow{2}{*}{8} & \multirow{2}{*}{$\mathrm{F}$} & $\mathbf{R}$ & 2 & \multirow{2}{*}{2} & 3.54 & 5 & - & $3 !$ & $6 / 6$ & & \multirow{2}{*}{+} \\
\hline & & & $\mathbf{L}$ & 2 & & 3.54 & 5 & $\cdots$ & $3 !$ & $6 / 5$ & & \\
\hline \multirow{2}{*}{40} & \multirow{2}{*}{12} & \multirow{2}{*}{$\mathbf{M}$} & $\mathbf{L}$ & 3 & \multirow{2}{*}{4} & 6.54 & 4 & + & $3 !$ & $6 / 6$ & & \multirow{2}{*}{+} \\
\hline & & & $\mathbf{R}$ & 1 & & 7.54 & 4 & & $3 !$ & $6 / 6$ & & \\
\hline \multirow{2}{*}{41} & \multirow{2}{*}{12} & \multirow{2}{*}{$\mathbf{M}$} & $\mathbf{R}$ & 4 & \multirow{2}{*}{4} & 7.54 & 12 & - & $3 !$ & $6 / 6$ & & \multirow{2}{*}{ 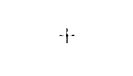 } \\
\hline & & & $\mathbf{L}$ & 2 & & 8.54 & 12 & - & $3 !$ & $6 / 6$ & & \\
\hline \multirow{2}{*}{46} & \multirow{2}{*}{6} & \multirow{2}{*}{$\mathrm{F}$} & $\mathbf{R}$ & 2 & \multirow{2}{*}{1} & 6.55 & 24 & $\cdots$ & 1 & $6 / 6$ & & \multirow{2}{*}{+} \\
\hline & & & $\mathbf{L}$ & 2 & & 6.55 & 24 & & 1 & $6 / 12$ & Faint & \\
\hline 50 & 23 & $\mathrm{~F}$ & $\mathbf{R}$ & 3 & & 10.56 & 4 & - & 1 & $6 / 6$ & & + \\
\hline
\end{tabular}

TABLE B

GROUP 2 CASES WITH A RELAPSING COURSE

\begin{tabular}{|c|c|c|c|c|c|c|c|c|c|c|c|c|}
\hline $\begin{array}{l}\text { Case } \\
\text { No. }\end{array}$ & $\begin{array}{l}\text { Age } \\
\text { (yrs) }\end{array}$ & Sex & $\begin{array}{c}\text { Eye } \\
\text { Involved }\end{array}$ & $\mid \begin{array}{c}\text { Duration } \\
\text { of } \\
\text { Symptoms } \\
\text { (wks) }\end{array}$ & $\begin{array}{l}\text { Interval } \\
\text { between } \\
\text { Onset in } \\
\text { Two Eyes } \\
\text { (wks) }\end{array}$ & $\begin{array}{c}\text { Date of } \\
\text { Starting } \\
\text { Treatment }\end{array}$ & $\begin{array}{c}\text { Duration } \\
\text { of } \\
\text { Treatment } \\
\text { (mths) }\end{array}$ & $\begin{array}{l}\text { Corneal } \\
\text { Vessels }\end{array}$ & $\begin{array}{c}\text { Interval between } \\
\text { Stopping } \\
\text { Treatment and } \\
\text { Assessment } \\
\text { (yrs) }\end{array}$ & $\begin{array}{c}\text { Visual } \\
\text { Acuity } \\
\text { (corrected) }\end{array}$ & $\begin{array}{l}\text { Corneal } \\
\text { Opacity }\end{array}$ & $\begin{array}{c}\text { Wassermann } \\
\text { Reaction }\end{array}$ \\
\hline \multirow{2}{*}{1} & \multirow{2}{*}{20} & \multirow{2}{*}{$\mathbf{F}$} & $\mathbf{R}$ & 4 & \multirow{2}{*}{4} & 1.52 & 66 & - & 1 & $6 / 9$ & Faint & \multirow{2}{*}{ t } \\
\hline & & & $\mathrm{L}$ & 1 & & 2.52 & 66 & - & 1 & $6 / 9$ & Faint & \\
\hline \multirow{2}{*}{4} & \multirow{2}{*}{19} & \multirow{2}{*}{$\mathrm{F}$} & $\mathbf{R}$ & 3 & \multirow{2}{*}{3} & 1.51 & 14 & + & 6 & $6 / 6$ & & \multirow{2}{*}{+} \\
\hline & & & $\mathrm{L}$ & 3 & & 2.51 & 1 & + & 7 & $6 / 6$ & & \\
\hline 6 & 11 & $\mathbf{M}$ & $\mathbf{R}$ & 1 & & 2.52 & 10 & + & 4 & $6 / 9$ & Faint & + \\
\hline \multirow{2}{*}{15} & \multirow{2}{*}{4} & \multirow{2}{*}{$\mathbf{F}$} & $\mathbf{R}$ & 1 & \multirow{2}{*}{2} & 3.52 & 6 & - & 4 & $6 / 36$ & & \multirow{2}{*}{+} \\
\hline & & & $\mathbf{L}$ & 1 & & 3.52 & 11 & - & 4 & $6 / 6$ & & \\
\hline \multirow{2}{*}{16} & \multirow{2}{*}{17} & \multirow{2}{*}{$\mathrm{F}$} & $\mathbf{R}$ & 12 & \multirow{2}{*}{4} & 4.52 & 18 & - & 3 & $6 / 6$ & & \multirow{2}{*}{+} \\
\hline & & & $\mathbf{L}$ & 4 & & 5.52 & 18 & - & 3 & $6 / 9$ & & \\
\hline \multirow{2}{*}{28} & \multirow{2}{*}{8} & \multirow{2}{*}{$\mathrm{F}$} & $\mathbf{L}$ & 2 & \multirow{2}{*}{4} & 4.53 & 20 & + & 3 & $6 / 9$ & & \multirow{2}{*}{+} \\
\hline & & & $\mathbf{R}$ & 1 & & 5.53 & 20 & + & 3 & $6 / 9$ & & \\
\hline 47 & 9 & $\mathbf{F}$ & $\mathbf{L}$ & 4 & & 7.55 & 8 & + & 2 & $6 / 9$ & & + \\
\hline \multirow{2}{*}{48} & \multirow{2}{*}{15} & \multirow{2}{*}{$\mathbf{F}$} & $\mathbf{R}$ & 1 & \multirow{2}{*}{2} & 7.55 & 12 & + & $1 \frac{1}{2}$ & $6 / 6$ & & \multirow{2}{*}{+} \\
\hline & & & $\mathbf{L}$ & 2 & & 7.55 & 12 & - & $1 \stackrel{5}{2}$ & $6 / 6$ & & \\
\hline \multirow{2}{*}{49} & \multirow{2}{*}{19} & \multirow{2}{*}{$\mathbf{F}$} & $\mathbf{L}$ & 2 & \multirow{2}{*}{4} & 10.55 & 18 & - & 1 & $6 / 5$ & Slight & + \\
\hline & & & $\mathbf{R}$ & 1 & & 11.55 & 17 & $\cdots$ & 1 & $6 / 6$ & Slight & + \\
\hline
\end{tabular}


TABLE C

GROUP 3. CASES RELAPSING WITHIN 3 MONTHS

\begin{tabular}{|c|c|c|c|c|c|c|c|c|c|c|c|c|}
\hline $\begin{array}{l}\text { Case } \\
\text { No. }\end{array}$ & $\begin{array}{l}\text { Age } \\
\text { (yrs) }\end{array}$ & Sex & $\begin{array}{c}\text { Eye } \\
\text { In- } \\
\text { volved }\end{array}$ & $\begin{array}{c}\text { Duration } \\
\text { of } \\
\text { Symptoms } \\
\text { (wks) }\end{array}$ & $\begin{array}{c}\text { Interval } \\
\text { between } \\
\text { Onset in } \\
\text { Two Eyes } \\
\text { (wks) }\end{array}$ & $\begin{array}{l}\text { Date } \\
\text { of } \\
\text { Onset }\end{array}$ & $\begin{array}{l}\text { Duration of } \\
\text { Treatment for } \\
\text { Each Relapse }\end{array}$ & $\begin{array}{l}\text { Corneal } \\
\text { Vessels }\end{array}$ & $\begin{array}{l}\text { Interval between } \\
\text { Stopping } \\
\text { Treatment and } \\
\text { Assessment } \\
\text { (yrs) }\end{array}$ & $\begin{array}{c}\text { Visual } \\
\text { Acuity } \\
\text { (corrected) }\end{array}$ & $\begin{array}{l}\text { Corneal } \\
\text { Opacity }\end{array}$ & $\begin{array}{c}\text { Wassermann } \\
\text { Reaction }\end{array}$ \\
\hline \multirow{2}{*}{2} & \multirow{2}{*}{18} & \multirow{2}{*}{$\mathbf{M}$} & $\mathbf{R}$ & 8 & & 2.51 & $\begin{array}{l}\text { Several over } \\
12 \text { months }\end{array}$ & + & 6 & $6 / 6$ & & \multirow{2}{*}{+} \\
\hline & & & $\mathrm{L}$ & 3 & & 2.51 & $\begin{array}{ll}\text { (1) } & 2.51 \text { to } 3.51 \\
\text { (2) } & 3.51 \text { to } 12.51 \\
\text { (3) } & 5.53 \text { to } 8.55\end{array}$ & + & $2 !$ & $6 / 6$ & & \\
\hline 21 & 9 & $\mathbf{M}$ & $\mathbf{L}$ & 3 & & 9.52 & $\begin{array}{ll}\text { (1) } & 9.52 \text { to } 12.52 \\
\text { (2) } & 1.53 \text { to } 4.53\end{array}$ & - & 4 & $6 / 6$ & & + \\
\hline \multirow{2}{*}{37} & \multirow{2}{*}{11} & \multirow{2}{*}{$\mathbf{F}$} & $\mathbf{R}$ & 2 & & 5.54 & (1) 5.54 to 7.54 & - & 3 & $6 / 9$ & & \multirow{2}{*}{+} \\
\hline & & & $\mathbf{L}$ & 2 & & 5.54 & (2) 8.54 to 12.54 & - & 3 & $6 / 9$ & & \\
\hline \multirow[t]{2}{*}{38} & \multirow[t]{2}{*}{9} & \multirow[t]{2}{*}{$\mathbf{M}$} & $\mathbf{R}$ & 1 & \multirow{2}{*}{1} & 5.54 & $\begin{array}{ll}\text { (1) } 5.54 \text { to } 6.54 \\
\text { (2) } 7.54 \text { to } 8.54\end{array}$ & - & $3 \frac{1}{2}$ & $6 / 6$ & & \multirow{2}{*}{+} \\
\hline & & & $\mathbf{L}$ & 1 & & 5.54 & (1) 5.54 to 6.54 & - & 3 & $6 / 6$ & & \\
\hline \multirow[t]{2}{*}{43} & \multirow[t]{2}{*}{5} & \multirow[t]{2}{*}{$\mathbf{F}$} & $\mathbf{R}$ & 2 & \multirow{2}{*}{2} & 8.54 & $\begin{array}{l}\text { (1) } 8.54 \text { to } 9.54 \\
\text { (2) } 11.54 \text { to } 12.54\end{array}$ & - & 3 & $6 / 6$ & & \multirow{2}{*}{+} \\
\hline & & & $\mathbf{L}$ & 2 & & 8.54 & (1) 8.54 to 9.54 & & $3 \frac{1}{2}$ & $6 / 6$ & & \\
\hline 45 & 19 & $\mathbf{F}$ & $\mathbf{L}$ & 8 & & 12.55 & $\begin{array}{l}\text { (1) } 12.55 \text { to } 1.56 \\
\text { (2) } 2.56 \text { to } 12.56\end{array}$ & & 1 & & & + \\
\hline
\end{tabular}

TABLE D

GROUP 4: 5 CASES RELAPSING AFTER MORE THAN 3 MONTHS

\begin{tabular}{|c|c|c|c|c|c|c|c|c|c|c|c|c|}
\hline $\begin{array}{l}\text { Case } \\
\text { No. }\end{array}$ & $\begin{array}{l}\text { Age } \\
\text { (yrs) }\end{array}$ & Sex & $\begin{array}{c}\text { Eye } \\
\text { In- } \\
\text { volved }\end{array}$ & $\begin{array}{c}\text { Duration } \\
\text { of } \\
\text { Symptoms } \\
\text { (wks) }\end{array}$ & $\begin{array}{l}\text { Interval } \\
\text { between } \\
\text { Onset in } \\
\text { Two Eyes } \\
\text { (wks) }\end{array}$ & $\begin{array}{l}\text { Date } \\
\text { of } \\
\text { Onset }\end{array}$ & $\begin{array}{l}\text { Duration of } \\
\text { Treatment for } \\
\text { Each Relapse }\end{array}$ & $\begin{array}{l}\text { Corneal } \\
\text { Vessels }\end{array}$ & $\begin{array}{l}\text { Interval between } \\
\text { Stopping } \\
\text { Treatment and } \\
\text { Assessment } \\
\text { (yrs) }\end{array}$ & $\begin{array}{c}\text { Visual } \\
\text { Acuity } \\
\text { (corrected) }\end{array}$ & $\begin{array}{l}\text { Corneal } \\
\text { Opacity }\end{array}$ & $\begin{array}{c}\text { Wassermann } \\
\text { Reaction }\end{array}$ \\
\hline \multirow{2}{*}{11} & \multirow{2}{*}{8} & \multirow{2}{*}{$\mathbf{M}$} & $\mathbf{R}$ & 5 & \multirow[b]{2}{*}{4} & 1.52 & $\begin{array}{l}\text { (1) } 1.52 \text { to } 3.52 \\
\text { (2) } 5.52 \text { to } 7.52 \\
\text { (3) } 10.52 \text { to } 10.53\end{array}$ & + & $2 \frac{1}{2}$ & $6 / 6$ & & \multirow{2}{*}{+} \\
\hline & & & $\mathbf{L}$ & 4 & & 2.52 & (1) 2.52 to 10.53 & Nil & $2 \frac{1}{2}$ & $6 / 6$ & Nil & \\
\hline \multirow{2}{*}{14} & \multirow{2}{*}{8} & \multirow{2}{*}{$\mathbf{F}$} & $\mathbf{R}$ & 3 & \multirow{2}{*}{ Nil } & 2.52 & $\begin{array}{l}\text { (1) } 2.52 \text { to } 4.52 \\
\text { (2) } 9.52 \text { to } 8.53\end{array}$ & Nil & $4 \frac{1}{2}$ & $6 / 6$ & Nil & \multirow{2}{*}{+} \\
\hline & & & $\mathbf{L}$ & 3 & & 2.52 & (1) 2.52 to 4.52 & + & 5 & $6 / 6$ & Nil & \\
\hline \multirow{2}{*}{17} & \multirow{2}{*}{28} & \multirow{2}{*}{$\mathbf{F}$} & $\mathbf{L}$ & 3 & \multirow{2}{*}{8} & 5.52 & $\begin{array}{l}\text { (1) } 5.52 \text { to } 8.52 \\
\text { (2) } 1.54 \text { to } 4.54\end{array}$ & Nil & 4 & $6 / 6$ & Nil & \multirow{2}{*}{+} \\
\hline & & & $\mathbf{R}$ & 2 & & 7.52 & $\begin{array}{l}\text { (1) } 7.52 \text { to } 10.52 \\
\text { (2) } 8.52 \text { to } 11.55\end{array}$ & Nil & 2 & $6 / 6$ & Nil & \\
\hline \multirow{2}{*}{18} & \multirow{2}{*}{15} & \multirow{2}{*}{$\mathrm{F}$} & L & 5 & \multirow{2}{*}{$10 \mathrm{mths}$} & 5.52 & $\begin{array}{l}\text { (1) } 5.52 \text { to } 3.53 \\
\text { (2) } 2.57 \text { to } 8.57\end{array}$ & Nil & i & $6 / 6$ & Nil & \multirow{2}{*}{+} \\
\hline & & & $\mathbf{R}$ & 4 & & 3.53 & (1) 3.52 to 4.54 & Nil & 4 & $6 / 6$ & Nil & \\
\hline \multirow{2}{*}{32} & \multirow{2}{*}{14} & \multirow{2}{*}{$\mathbf{F}$} & $\mathbf{L}$ & 3 & \multirow{2}{*}{2} & 10.53 & $\begin{array}{l}\text { (1) } 10.52 \text { to } 12.53 \\
\text { (2) } 12.54 \text { to } 4.55\end{array}$ & + & 3 & $6 / 6$ & Nil & \multirow{2}{*}{+} \\
\hline & & & $\mathbf{R}$ & 1 & & 10.52 & (1) 10.52 to 12.52 & Nil & 5 & $6 / 6$ & Nil & \\
\hline
\end{tabular}


TABle E

GROUP 5: 4 DEFAULTERS

\begin{tabular}{|c|c|c|c|c|c|c|c|c|c|}
\hline $\begin{array}{l}\text { Case } \\
\text { No. }\end{array}$ & $\begin{array}{r}\text { Age } \\
\text { (yrs) }\end{array}$ & Sex & $\begin{array}{c}\text { Eye } \\
\text { Involved }\end{array}$ & $\begin{array}{c}\text { Duration } \\
\text { of } \\
\text { Symptoms } \\
\text { (wks) }\end{array}$ & $\begin{array}{l}\text { Interval } \\
\text { between } \\
\text { Onset in } \\
\text { Two Eyes } \\
\text { (wks) }\end{array}$ & $\begin{array}{c}\text { Date of } \\
\text { Starting } \\
\text { Treatment }\end{array}$ & $\begin{array}{l}\text { Duration } \\
\text { of } \\
\text { Treatment } \\
\text { (mths) }\end{array}$ & $\begin{array}{l}\text { Presence } \\
\text { of } \\
\text { Corneal } \\
\text { Vessels }\end{array}$ & Remarks \\
\hline 29 & 14 & $\mathbf{F}$ & L & 8 & & 5.53 & 6 & Nil & $\begin{array}{l}\text { Good immediate control-maintained } \\
\text { for } 6 \mathrm{mths}\end{array}$ \\
\hline 30 & 30 & $\mathbf{F}$ & $\mathbf{L}$ & 3 & & 7.53 & 2 & + & $\begin{array}{l}\text { Attended } 4 \text { wks after ceasing treat- } \\
\text { ment then defaulted }\end{array}$ \\
\hline \multirow{2}{*}{42} & \multirow{2}{*}{10} & \multirow{2}{*}{$\mathbf{F}$} & $\mathbf{R}$ & 5 & & 10.54 & 17 & + & \multirow{2}{*}{$\begin{array}{l}\text { Defaulted January, } 1956 \\
\text { Visual Acuity } R \text {. eye } 3 / 60 \text {, L. eye } 6 / 18 \\
\text { Irregular attendance during treat- } \\
\text { ment } \\
\text { Recurrent relapses }\end{array}$} \\
\hline & & & $\mathbf{L}$ & 1 & 4 & 11.54 & 17 & Nil & \\
\hline \multirow{2}{*}{44} & \multirow{2}{*}{16} & \multirow{2}{*}{$\mathbf{M}$} & $\mathbf{R}$ & 3 & \multirow{2}{*}{8} & 10.54 & 12 & + & \multirow{2}{*}{$\begin{array}{l}\text { Good immediate response main- } \\
\text { tained for } 12 \mathrm{mths} \text { then defaulted }\end{array}$} \\
\hline & & & $\mathbf{L}$ & 1 & & 12.54 & 12 & + & \\
\hline
\end{tabular}

TABLE F

GROUP 6: 2 CASES DEVELOPING CHOROIDITIS

\begin{tabular}{|c|c|c|c|c|c|c|c|c|c|c|c|c|c|}
\hline $\begin{array}{l}\text { Case } \\
\text { No. }\end{array}$ & $\begin{array}{c}\text { Age } \\
\text { (yrs) }\end{array}$ & Sex & $\begin{array}{c}\text { Eye } \\
\text { In- } \\
\text { volved }\end{array}$ & $\begin{array}{c}\text { Duration } \\
\text { of } \\
\text { Symptoms } \\
\text { (wks) }\end{array}$ & $\begin{array}{c}\text { Interval } \\
\text { between } \\
\text { Onset in } \\
\text { TwoEyes } \\
\text { (wks) }\end{array}$ & $\begin{array}{c}\text { Date of } \\
\text { Starting } \\
\text { Treat- } \\
\text { ment }\end{array}$ & $\begin{array}{l}\text { Corneal } \\
\text { Vessels }\end{array}$ & $\begin{array}{c}\text { Duration } \\
\text { of } \\
\text { Treat- } \\
\text { ment } \\
\text { (mths) }\end{array}$ & $\begin{array}{c}\text { Interval } \\
\text { between } \\
\text { Stopping } \\
\text { Treatment } \\
\text { and } \\
\text { Assessment } \\
\text { (yrs) }\end{array}$ & $\begin{array}{l}\text { Visual } \\
\text { Acuity }\end{array}$ & $\begin{array}{l}\text { Corneal } \\
\text { Opacity }\end{array}$ & $\begin{array}{c}\text { Wasser- } \\
\text { mann } \\
\text { Reaction }\end{array}$ & Remarks \\
\hline \multirow{2}{*}{27} & \multirow{2}{*}{14} & \multirow{2}{*}{$\mathbf{F}$} & L & 4 & \multirow{2}{*}{15} & 10.52 & + & 12 & 4 & $6 / 18$ & Nil & \multirow{2}{*}{+} & \multirow{2}{*}{$\begin{array}{l}\text { Diffuse } \\
\text { choroiditis } \\
\text { in both eyes }\end{array}$} \\
\hline & & & $\mathbf{R}$ & 1 & & 3.53 & Nil & 7 & 4 & $6 / 60$ & Nil & & \\
\hline \multirow{2}{*}{34} & \multirow{2}{*}{9} & \multirow{2}{*}{$\mathbf{F}$} & L & 3 & \multirow{2}{*}{4} & 12.53 & Nil & 12 & 3 & $6 / 60$ & Nil & \multirow{2}{*}{+} & \multirow{2}{*}{$\begin{array}{l}\text { Diffuse } \\
\text { choroiditis } \\
\text { in both eyes }\end{array}$} \\
\hline & & & $\mathbf{R}$ & 2 & & 1.54 & Nil & 12 & 3 & $4 / 60$ & Nil & & \\
\hline
\end{tabular}

